\title{
Discovery of Known and Novel Viral Genomes in Soybean Aphid by Deep Sequencing
}

\author{
Ying Feng, Bioinformatics and Computational Biology Program and Plant Pathology and Microbiology Department, \\ lowa State University, Ames 50011; Elizabeth N. Krueger, Plant Pathology and Microbiology Department, lowa \\ State University; Sijun Liu, Entomology Department, lowa State University; Karin Dorman, Bioinformatics and \\ Computational Biology Program and Statistics Department, lowa State University; Bryony C. Bonning, Entomology \\ Department, lowa State University; and W. Allen Miller, Bioinformatics and Computational Biology Program \\ and Plant Pathology and Microbiology Department, lowa State University
}

Accepted for publication 14 February 2017.

\section{ABSTRACT}

The phytobiome includes not just cellular microorganisms, but also all viruses associated with plants: the virome. Plants and aphids exchange viruses regularly and efficiently; thus, the plant virome is tightly linked with the aphid virome. Yet, little is known about aphid viromes, particularly that of the soybean aphid (Aphis glycines), one of the most economically important pest insects of soybean. To sample the soybean aphid virome, and to seek new viruses as potential biological control agents, we employed next-generation sequencing. Genomes isolated from viruses in soybean aphids collected at four sites revealed many viruses, and six complete or nearly complete genomes were assembled. Most abundant were the picornavirus-like dicistroviruses Aphid lethal paralysis virus and Rhopalosiphum padi virus. We also sequenced the genome of a new dicistrovirus, Big
Sioux River virus, fragments of which had been found previously in honey bee. Genome sequences that represent two entirely new virus families were obtained. These include an abundant tetravirus-like virus and a virus distantly related to cileviruses of plants and negeviruses of insects. Surprisingly, Cotton leafroll dwarf virus, a member of the genus Polerovirus, was found in soybean aphids from China, suggesting that the soybean aphid may be a vector of this virus. This virus had not been reported previously in China or in soybean. This study provides a peek into the rapidly expanding, largely unexplored world of insect viromes that will provide valuable knowledge for future understanding of plant-virus-vector interactions.

Additional keywords: Aphis glycines virus 2, honey bee viruses.
The viral component of the plant phytobiome, the plant virome, is influenced heavily by insect vectors, as most plant viruses are transmitted by insects. Aphids are the most common vectors of plant viruses ( $\mathrm{Ng}$ and Falk 2006); thus, via its effects on the plant virome and on aphid fitness, the aphid virome affects the competitiveness of plants in natural and agricultural ecosystems (Lacroix et al. 2014; Malmstrom et al. 2005; Mordecai et al. 2016). The aphid virome includes (i) insect viruses that infect aphids and possibly other insects, (ii) plant viruses that replicate only in plants but are transmitted by

Corresponding author: W. A. Miller; E-mail address: wamiller@iastate.edu

Author contributions. W.A.M. and B.C.B. conceived the study. E.N.K. isolated virus and RNA for sequencing. E.N.K. and Y.F. assembled the sequences. Y.F., S.L., K.D., and W.A.M. analyzed the data. Y.F., B.C.B., and W.A.M. wrote the manuscript.

*The $e$-Xtra logo stands for "electronic extra" and indicates that one supplementary figure and three supplementary tables are published online.

(c) (i) $($ ) $\odot$ Copyright (C) 2017 The Author(s). This is an open access article distributed under the CC BY-NC-ND 4.0 International license. aphids, (iii) viruses that replicate in aphids and in plants, (iv) viruses of symbiotic and parasitic organisms in aphids, and (v) viruses that associate with aphids with no apparent biological role. Plants serve as reservoirs (i.e., immobile vectors) for viruses that infect aphids (Gildow and D'Arcy 1988). Aphid viruses can reduce aphid fecundity and lifespan (D'Arcy et al. 1981; Laubscher and von Wechmar 1992, 1993) and thus reduce damage to plants by aphid feeding or transmission of plant viruses. Moreover, plant viruses can either hinder (Cassone et al. 2014; Donaldson and Gratton 2007; Fiebig et al. 2004; McIntyre et al. 1981; Mowry 1994) or benefit (Belliure et al. 2008; Casteel et al. 2014) their insect vectors. To begin to understand this complex interplay between plant and aphid viromes, it is essential to know which viruses are present in aphids. Thus, here we determine viromes of soybean aphid (Aphis glycines) from four geographically distinct locations.

The soybean aphid, endemic in China, arrived in the Midwestern United States in 2000, where it quickly became the dominant insect pest of soybean (Ragsdale et al. 2004). Populations of soybean aphid increase rapidly, causing heavy infestations that lead to significant yield loss. The soybean aphid has been estimated to cost up to U.S. \$3.6 billion annually in losses and control costs (Kim 
et al. 2008). It damages plants directly (Macedo et al. 2003) and produces sugar-rich honeydew, which makes plants sticky, difficult to handle, and fosters growth of sooty mold that further reduces yield. In addition to this damage, the soybean aphid is a vector of, among others, Alfalfa mosaic virus (AMV) (Bol 2003), Soybean mosaic virus (SMV) (Jayaram et al. 1991), and (inefficiently) Soybean dwarf virus (SbDV) (Damsteegt et al. 2011). These viruses do not replicate in the aphid. Only a recently discovered novel RNA virus, Aphis glycines virus 2 (AGV2), is known to replicate in soybean aphid (Liu et al. 2016). An unrelated viral sequence called Aphis glycines virus 1 (AGV1), isolated from soybean aphid, has been deposited in GenBank (accession number KF360262), but whether this RNA actually infects soybean aphid has not been reported.

Several viruses are known to infect other aphid species. These include Rhopalosiphum padi virus (RhPV) and Aphid lethal paralysis virus (ALPV), in the family Dicistroviridae, order Picornavirales. Dicistroviruses differ from other members of the Picornavirales of animals in that they encode two open reading frames (ORFs) instead of one (Bonning and Miller 2010). ORF1 encodes nonstructural genes, and ORF2, which is translated via a novel internal ribosome entry site (Costantino et al. 2008), encodes the virion proteins. As for all picorna-like viruses, the functional proteins are cleaved from the translated polyprotein. RhPV is known to infect aphids of cereals (Gildow and D'Arcy 1990). It circulates (without replicating) in the plant phloem and is transmitted from plants to uninfected aphids (Gildow and D'Arcy 1988). ALPV was first isolated from $R$. padi after observation of infected aphids moving away from the food source and death induced by paralysis (Laubscher and von Wechmar 1993; Williamson et al. 1988). Several ALPV-like viruses have been identified in organisms other than aphids, such as honey bees, and also in bat fecal samples (Dombrovsky and Luria 2013; Ge et al. 2012; Granberg et al. 2013; Liu et al. 2014; Runckel et al. 2011). ALPV is transmitted vertically in $R$. padi and the English grain aphid (Sitobion avenae) (Laubscher and von Wechmar 1992).

Other aphid viruses include the following: (i) Brevicoryne brassicae virus of the family Iflaviridae (Picornavirales), which infects cabbage aphid (Brevicoryne brassicae) (Ryabov 2007), (ii) a virus resembling those in the family Flaviviridae, but with a genome twice as large, in Macrosiphum euphorbiae (Teixeira et al. 2016), (iii) a pea aphid virus (Acyrthosiphon pisum virus) (van den Heuvel et al. 1997) distantly related to the Picornaviridae; and (iv) a rosy apple aphid virus (Dysaphis plantaginea, Rosy apple aphid virus) (Ryabov et al. 2009), distantly related to the family Caliciviridae. Small DNA viruses in the genus Densovirus (Parvoviridae) have been discovered in Acyrthosiphon pisum, D. plantaginea (Ryabov et al. 2009), and Myzus persicae (van Munster et al. 2003). All of these viruses have small, icosahedral virions.

By far, the most viruses known to be associated with aphids are plant viruses. Hundreds of plant viruses are transmitted by aphids (Brault et al. 2010). They may bind transiently to mouthparts for just minutes (Ng and Falk 2006) or circulate within the aphid for the life of the aphid (Gray and Gildow 2003). The vast majority of aphid-vectored plant viruses do not replicate within the aphid. However, plant viruses in the family Rhabdoviridae also replicate in their insect vectors, which include aphids (Mann and Dietzgen 2014). Thus, these rhabdoviruses can be considered viruses of aphids as well as plant viruses. However, no rhabdoviruses are known that replicate in the soybean aphid.

By allowing the study of multiple virus populations, deep sequencing has been the most unbiased, and cost- and time-efficient way to identify and classify novel and known viruses, viral diversity and evolution, and transmission (Liu et al. 2011). To investigate which, if any, viruses are present in soybean aphids, we sequenced total RNA from partially purified virus preparations from colonies of soybean aphids that were originally collected in Iowa, Michigan, and Ohio, and from a sample of dead aphids from China. After de novo assembly and reference mapping assembly, we obtained complete or nearly complete genome sequences of (i) a novel, ubiquitous tetravirus-like virus, (ii) dicistroviruses nearly identical to or similar to those known to infect other aphid species, (iii) a virus, of which fragments had been found previously in honey bees, (iv) a plant polerovirus, and (v) a new cilevirus-like virus of unknown host. Several of the sequences were highly divergent from previously isolated or discovered viruses.

\section{MATERIALS AND METHODS}

Aphids. Aphid handling from the various samples was conducted on separate days to avoid cross-contamination between the samples. The aphid colonies were maintained and isolated from each other spatially and temporally.

The four samples characterized were obtained from Ohio State University, Wooster, OH; Michigan State University, East Lansing, MI; Iowa State University, Ames, IA; and Wuhan University, Wuhan, China. The aphids from China were not frozen at the time of collection or during international shipping, but were maintained at $-80^{\circ} \mathrm{C}$ upon arrival in the United States. The Ohio and Michigan samples were maintained in colonies for at least 2 years in separate growth chambers before virus isolation. The Iowa sample is a lab colony annually supplemented with field-collected soybean aphid.

Sample collection, virus purification, and viral RNA extraction. Two heavily infested trifoliate leaves and stems were collected from a colony for each U.S. location. The trifoliates were placed into a container at $-20^{\circ} \mathrm{C}$ for at least $1 \mathrm{~h}$ to kill the aphids. The aphids were then isolated from the trifoliates by using a fresh fine-tipped paintbrush to move the aphids from the leaves into a microcentrifuge tube. A new paintbrush was used for each sample to minimize the chance of crosscontaminating the samples. Once harvested, the aphids were maintained at $-80^{\circ} \mathrm{C}$ until the RNA extraction.

We partially purified virus particles (virions) by differential centrifugation. Soybean aphids were ground in liquid nitrogen, suspended in $15 \mathrm{ml}$ of $0.01 \mathrm{M}$ sodium phosphate buffer, $\mathrm{pH} 7.0$, and $7.5 \mathrm{ml}$ of chloroform. All subsequent steps were performed on ice or at $4{ }^{\circ} \mathrm{C}$. After vigorous mixing, the suspension was centrifuged at $7,649 \times g$ for $25 \mathrm{~min}$. The supernatant was then centrifuged at $105,638 \times g$ for $6.5 \mathrm{~h}$ The pellet was resuspended in $5 \mathrm{ml}$ of phosphate buffer, clarified by centrifugation in $5 \times 1.5 \mathrm{ml}$ microcentrifuge tubes $(12,000 \times g)$. This supernatant was layered onto a $0.5-\mathrm{ml} 30 \%$ sucrose cushion in $1.5-\mathrm{ml}$ ultracentrifuge tubes. These were subjected to centrifugation at 45,000 rpm $(124,858 \times g)$ for $3 \mathrm{~h}$ in a Sorvall S45A rotor in a Sorvall M150 ultracentrifuge. The pellet was resuspended in a small volume of phosphate buffer and clarified by brief microcentrifugation to remove any insoluble material. Particles were treated with ribonuclease to eliminate the nonencapsidated (nonviral) RNAs and then treated with Proteinase K to destroy the ribonuclease activity and remove the capsid protein. RNA was extracted using TRIzol Reagent (Life Technologies, Grand Island, NY) according to the manufacturer's protocol. The Trizol was heated to $65^{\circ} \mathrm{C}$ and the initial 5-min incubation was also carried out at $65^{\circ} \mathrm{C}$. The resultant RNA was salt/ethanol precipitated, resuspended in ultrapure distilled water, and analyzed on a Nanodrop instrument.

Library preparation and sequencing. The RNA samples were submitted for library preparation at the Iowa State University DNA Facility using the Illumina TruSeq library preparation kit beginning with the fractionation and adapter ligation without the polyA selection step. Then the samples were run in a single lane on the Illumina HiSEquation 2000 for 100-bp paired-end sequencing. The data were returned as four files for the China, Michigan, Ohio, and Iowa samples.

Sequence assembly and full virus genome discovery. The files were imported into the CLC Genomics Workbench version 6.0.4 (CLC Bio, Boston, MA) as Illumina paired-end reads, discarding 
the read names but maintaining the quality scores. For quality trimming, we used the default quality cutoff value 0.05 and the maximum number of ambiguities as 2 per 100-bp read to process each sample. For adapter trimming, we removed adapters from the Illumina adapter list. For sequence filtering, we filtered the reads below length 15 or above length 108. The output files were saved within the CLC database. Supplementary Table S1 gives the summary statistics of the data before and after trimming for each input file, including the number of reads, average length of the reads, number of reads retained after trimming, the percentage of the input reads that are retained, and average length after trimming.

The paired trimmed sequence data were assembled de novo using the CLC Genomics Workbench to form preliminary contigs. CLC's de novo assembly works by using de Bruijn graphs. The approach is to make a table of all subsequences of a certain length (called words) found in the reads. The word size is determined automatically in CLC. When there is an SNP or a sequencing error, the de Bruijn graph includes a so-called bubble. Bubbles less than a certain size are collapsed by removing the less commonly observed variant. In this study, we used the default bubble size, 50. We also used the default value $200 \mathrm{bp}$ for the minimum contig length. To assess the quality of the assembly, we performed scaffolding using CLC and compared the estimated insert size distribution with the library construction method. After assembly, we selected the option to map reads back to the preliminary contigs using default parameters: 2 for mismatch cost, 3 for insertion cost, 2 for deletion cost, 0.5 for length fraction, and 0.8 for similarity fraction. Supplementary Table S2 gives summary statistics of the preliminary contigs, including the number of contigs, average length of contigs, and statistic of $\mathrm{N} 25$, $\mathrm{N} 50$, and N75 (e.g., N25 is the length of the smallest contig in the set of largest contigs that add up to $25 \%$ of the total added contig lengths). Supplementary Figure S1 provides the estimated insert size distribution.

The preliminary contigs included genomic fragments from many organisms. To identify likely virus contigs, the contigs were queried against the NCBI nucleotide and protein database using BLASTn and BLASTx using default parameters in the CLC workbench. The filter parameters for BLAST used default value. Contigs with significant hits (E-value $<1 \mathrm{E}-03$ for BLASTx, E-value $<1 \mathrm{E}-10$ for BLASTn) to NCBI sequences with the word "virus" in the description were isolated as putative viral contigs (Table 1). The following steps were then run in parallel for four sets of preliminary contigs: putative viral contigs identified by BLASTx hit, putative viral contigs identified by BLASTn hit, contigs with a BLASTx hit, and contigs with a BLASTn hit. The four sets were reassembled to generate long contigs by de novo assembly as described previously, resulting in four sets of long contigs: long BLASTx viral contigs, long BLASTn viral contigs, long BLASTx contigs, and long BLASTn contigs. We call the union of the long BLASTx and BLASTn viral contigs "long viral contigs". These long viral contigs were queried against the all-organism NCBI database using
BLASTx and BLASTn with default parameters. Those long viral contigs that matched to viruses with E-value $<1 \mathrm{E}-05$ were retained (Supplementary Table S3). We call long viral contigs that have hits by either BLASTx or BLASTn "retained long viral contigs". Finally, all retained long viral contigs were BLASTED using default parameters against an in-house database consisting of all long BLASTx contigs and long BLASTn contigs, to look for nonviral long contigs that overlap long viral contigs. This essential step identifies novel viral sequence as contigs, which may have only partial homology to known viral sequence. Finally, we assembled the putative virus genomes manually by checking the overlapping information from the final BLAST. This process assembled the long retained viral contigs into viral genomes. Once the putative viral genomes were assembled, the whole sequence was further characterized in CLC Genomics Workbench by determining the potential ORFs using default parameters.

Phylogenetic and conservation analyses. Multiple sequence alignment, percent identity calculation and phylogenetic inference were performed using MEGA, version 6, software (Tamura et al. 2011). Specifically, ClustalW produced multiple amino acid sequence alignments using default parameters. We chose to align the amino acid sequences of the RNA-dependent RNA polymerase (RdRp) for each virus and several related viruses identified by BLAST (BLAST searches described above). Phylogenetic trees were inferred using the maximum likelihood method with nearestneighbor-interchange and the default initial tree using 500 bootstrap replicates. We assume the Jones-Taylor-Thornton (JTT) model, uniform rates across sites, and complete deletion for gaps/missing data in the alignment.

\section{RESULTS}

Deep sequencing analysis of virus hits. In the HiSeq data used in this study, a total of $263,808,602$ reads were obtained: $18 \%$ from China, $26 \%$ from Iowa, $24 \%$ from Ohio, and $32 \%$ from the Michigan sample. After trimming, $0.9 \%$ of the reads were removed because they failed our analysis criteria, leaving between 47 million and 82 million reads per sample (Table 1). With initial de novo assembly, $64.2 \%$ of the raw reads were from contigs ranging in length from 157 to 27,554 bp (Fig. 1; Table 1). The reads distribution between the four samples as well as the summary statistics for the de novo assembly are reported in Supplementary Table S1. As shown in the workflow (Fig. 1), we performed BLASTn and BLASTx to identify contigs with potential viral sequences. In total, 25 and $17 \%$ of the reads analyzed by BLASTn and BLASTx, respectively, hit known viral gene sequences (Fig. 1). We define a hit as a near-match with e-value of 1E-03 for BLASTx, and 1E-10 for BLASTn as the cutoffs for significance. Most of the reads hit aphid genome sequences with e-value below $1 \mathrm{E}-60$. We obtained longer contigs by reassembling the potential viral contigs.

TABLE 1

Summary of sequencing results by location

\begin{tabular}{|c|c|c|c|c|c|c|c|}
\hline \multirow[b]{2}{*}{$\begin{array}{l}\text { Sample } \\
\text { location }\end{array}$} & \multirow[b]{2}{*}{$\begin{array}{l}\text { Reads after } \\
\text { trimming }\end{array}$} & \multirow[b]{2}{*}{$\begin{array}{c}\text { Number of } \\
\text { preliminary contigs }\end{array}$} & \multirow{2}{*}{$\begin{array}{l}\text { Average length of } \\
\text { preliminary } \\
\text { contigs (bp) }\end{array}$} & \multicolumn{2}{|c|}{ 1st BLASTn } & \multicolumn{2}{|c|}{ 1st BLASTx } \\
\hline & & & & $\begin{array}{l}\text { Number of } \\
\text { viral contigs }\end{array}$ & $\begin{array}{l}\text { Average } \\
\text { length }\end{array}$ & $\begin{array}{l}\text { Number of } \\
\text { viral contigs }\end{array}$ & $\begin{array}{l}\text { Average } \\
\text { length }\end{array}$ \\
\hline lowa & $69,028,800$ & 30,997 & 546 & 173 & 715 & 52 & 583 \\
\hline Michigan & $82,248,632$ & 35,065 & 525 & 1,230 & 509 & 81 & 514 \\
\hline
\end{tabular}


de novo assembly. From the de novo assembly, 4,932, 30,996, 35,065 , and 27,512 contigs were generated in the samples from China, Iowa, Michigan, and Ohio, respectively (Table 1). A large number of the contigs were bacterial or insect sequences. For example, nearly $66 \%(20,164)$ of contigs were identified as insect in the sample from Iowa. Merely $2.6 \%$ of the de novo assembled, BLAST identified contigs were determined to be of viral origin. These sequences had similarity to members of the families Caulimoviridae, Dicistroviridae, Herpesviridae, Iflaviridae, Luteoviridae, Mimiviridae, Nodaviridae, Permutotetraviridae, and Virgaviridae.

ALPV, RhPV, and sequence fragments called Big Sioux River virus (BSRV) were the most common virus hits by BLAST for the contigs in all four samples. However, most of the viral contigs differed among the regions. Several long viral contigs ( $>1,000 \mathrm{bp})$ identified as close to Cotton leafroll dwarf virus (CLRDV) and Maize yellow dwarf virus were found only in the China sample. Several long contigs matching Drosophila A virus and Kashmir bee virus were found in the Iowa and Michigan samples based on both results of BLASTn and BLASTx. A 949-nt contig in the Ohio sample was identified as distantly related to Citrus leprosis virus cytoplasmic type 2 (CiLV-C2).

Genome assembly of known and novel viruses. Six contig assemblies, that we call segments, were obtained ranging from 4,839 to $10,174 \mathrm{nt}$ (Table 2). Of the six segments, three (S2, S4, and S5) were closely related to ALPV-AP1, RhPV, and CLRDV, with 88, 93.7, and 93\% full-length amino acid sequence identities, respectively. These three segments had lengths, and ORF arrangements, similar to those of the identified related virus genomes.

The other three segments (S1, S3, and S6) shared low amino acid identity with the top BLASTx hits. S1 is the genome of AGV2 which is described in detail by Liu et al. (2016). The top BLAST hit for S1 (AGV2) was Euprosterna elaeasa virus (EeV) with $33 \%$ amino acid identity in ORF1. S1 was found in aphids from the United States with abundant $(22,194$ to 35,895$)$ read counts (Table 2). From the Ohio sample, we assembled a 10,147-nt segment named S3, which has $34 \%$ amino acid identity with CiLV-C2. This segment is similarly related to Negev virus, Loreto virus, and Hibiscus green spot virus with $\sim 33 \%$ amino acid identity to all three viruses. S6, with length of $9,445 \mathrm{nt}$, was most similar to RhPV. In a previous study of sequences isolated from honey bee, four contigs of size 1,473, 861, 1,164, and 1,311 nt (GenBank JF423195, JF423196, JF423197, and JF423198) were designated as belonging to a hypothetical BSRV after its place of discovery in South Dakota. Segment S6 contains sequences with high amino acid sequence identity to all four contigs attributed to BSRV (Fig. 2F).

Virus genome organizations. Segment S1 (AGV2) contains four ORFs (Fig. 2A) with sizes of 3,438 nt (ORF1), $510 \mathrm{nt}$ (ORF2), $735 \mathrm{nt}$ (ORF3), and $810 \mathrm{nt}$ (ORF4). Part of ORF1 had similarity to polymerases of $\mathrm{EeV}$ and Thosea asigna virus $(\mathrm{TaV})$, while ORF3 of S1 had highest similarity to the capsid proteins of two plant viruses: a sobemovirus found in bat feces and Pothos latent virus, an aureusvirus (Tombusviridae) (Fig. 2A). In the BLASTx analysis, the S2 sequence showed 95 and $94 \%$ amino acid sequence identity with ORF1 (GenBank AFU81560) and ORF2 (GenBank AFU81561), respectively, of ALPV-An (Fig. 2B).

Segment S3 contains four large ORFs (Fig. 2C). The top BLASTx hit of S3 is CiLV-C2, which is a bipartite RNA virus. RNA1 of CiLV-C2 (8,717 nt) contains two ORFs, and RNA2 (4,989 nt) contains five ORFs (Roy et al. 2013). ORF1 and ORF2 of S3 showed $33 \%$ amino acid sequence identity with the two ORFs in RNA1 of CiLV-C2, but ORFs 3 and 4 had no matches, and there was no match to RNA2 of CiLV-C2 anywhere in the sequence of S3 (Fig. 2C).

Segment S4 contains two ORFs (ORF1: nt 580 to 6,576; ORF2: nt 7,103 to 9,560 ) with around $90 \%$ sequence similarity to RhPV (Fig. 2D). S5 has the genome organization of its top BLAST hit, the polerovirus CLRDV, having seven ORFs (Fig. 2E). Based on similarity with other poleroviruses, ORFs $0,1,2,3 \mathrm{a}, 3,4$, and 5 of CLRDV are predicted to encode the silencing suppressor protein, protease-VPg, RdRp, long-distance movement protein, coat protein, movement protein, and read-through protein, respectively (Correa et al. 2005; Smirnova et al. 2015). The sequence of S6 has two ORFs (ORF1: nt 3 to 2,150; ORF2: nt 2,753 to 8,752) with 71 and $73 \%$ amino acid identity to the polyprotein (ABX74939) and structural polyprotein (ABX74940) of RhPV, respectively. In S6, we found regions of even greater similarity to all four contigs of BSRV (Fig. 2F).

Virus phylogenetic analysis. Based on ClustalW multiple alignments of the RdRp amino acid sequences, we constructed multiple phylogenetic trees (Fig. 3A). S1 (AGV2) is only distantly related to all top hitting viruses. In particular, $\mathrm{S} 1$ is adjacent to the branches
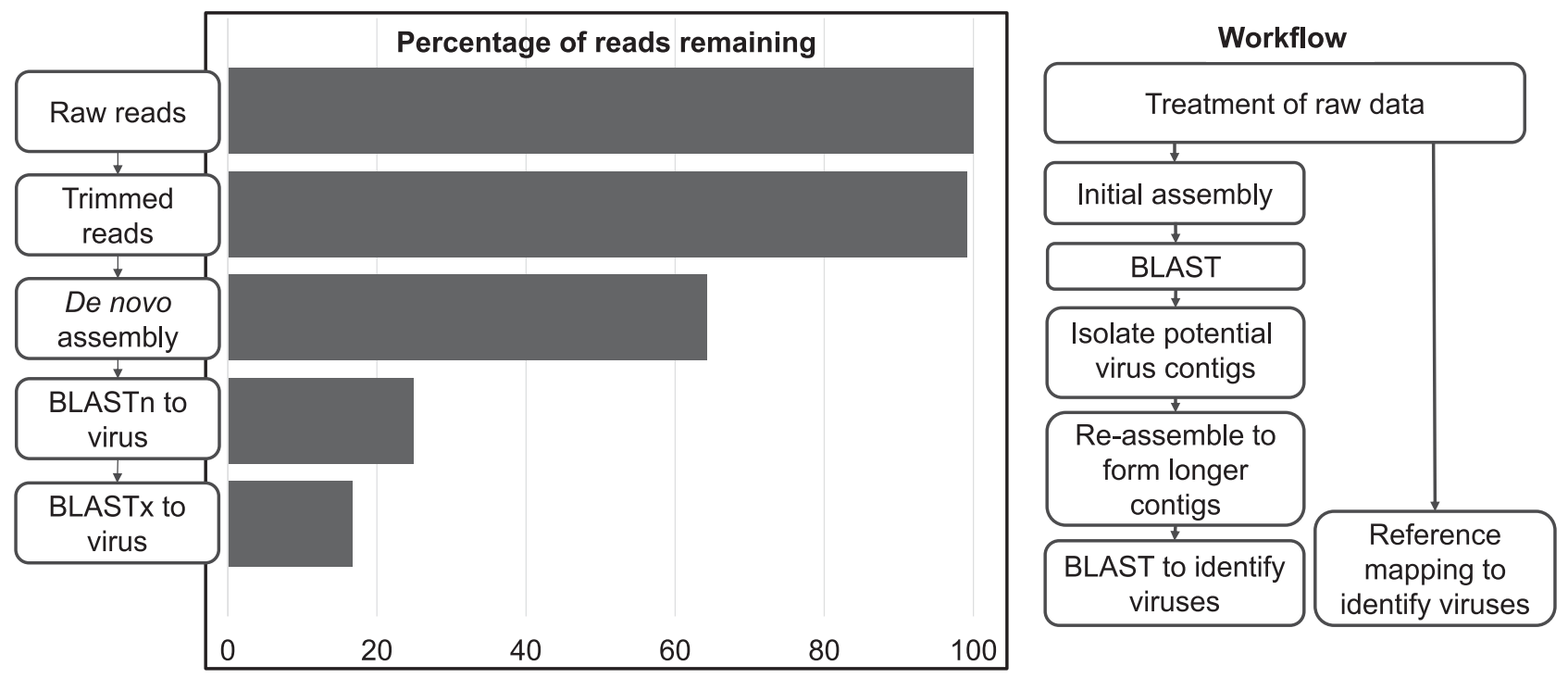

Fig. 1. Workflow for processing deep sequencing data. Two strategies used to analyze the data were de novo assembly and reference mapping. Average percent remaining reads after each of the filtering steps is plotted. Low-quality reads were removed first, followed by BLASTn and BLASTx comparison with virus sequences. 
of $\mathrm{EeV}$ and $\mathrm{TaV}$, which belong to family Permutotetraviridae, genus Alphapermutotetravirus. The other two top hitting viruses are unclassified. Drosophila A virus is an unclassified dsRNA virus, and Bat sobemovirus is an unclassified sobemovirus from bat feces (Figs. 2A and 3A).

Segments S2, S4, and S6 all have high identity to RhPV and ALPV. As already determined by BLASTx, S2 is most closely related to ALPV, while $\mathrm{S} 4$ is most closely related to RhPV (Fig. 3B). These six sequences as well as Drosophila $C$ virus and Cricket paralysis virus belong to the genus Cripavirus of the family Dicistroviridae.

A phylogenetic tree was constructed based on multiple alignments of the top BLASTx hits to the S3 sequence segment. S3 did not group closely to any virus in the phylogenetic tree (Fig. 3C). Its closest relatives are CiLV-C, and Lereto virus, Piura virus, Negewotan virus, and Negevirus, which are novel insect viruses, related distantly to CiLV-C (Vasilakis et al. 2013). The other family in this tree, Virgaviridae, includes only plant viruses, such as Tobacco mosaic virus.

Segment S5 is a sequence of a strain of CLRDV, with 93\% sequence identity, and belonging to the genus Polerovirus (Luteoviridae), most closely related to another polerovirus, Maize yellow dwarf virus-RMV (Fig. 3D). Other close relatives include the plant poleroviruses Melon aphid-born yellows virus and Pepper vein yellows virus.

\section{DISCUSSION}

Our study reveals genomes of known and novel viruses, all but one of which were not known to associate with soybean aphid. The geographically diverse sample sources allowed us to be more informed on the viruses the soybean aphids carry, and the deep sequencing allowed us to obtain sufficient reads to assemble reliable complete genomes in an unbiased way. The results provide valuable information on viral diversity and evolution.

Segment S1. S1 is the genome of a novel virus recently described as AGV2 (Liu et al. 2016). An unrelated sequence submitted previously to GenBank was named AGV1 (accession number KF360262). AGV2 was found in all samples of U.S. soybean aphids, and partial sequences were found in the Chinese sample, extending previous observations that no soybean aphids have been found without this virus (Liu et al. 2016). There were insufficient reads of AGV2 from the Chinese soybean aphid sample to assemble a genome, but overall this sample was highly enriched in other viral sequences, possibly because the sample was stored at room temperature, causing selective degradation of less stable viruses and nonviral RNA, whereas the Iowa, Michigan, and Ohio virions were isolated from live aphid colonies. AGV2 is difficult to purify, suggesting it has an unstable virion (Liu et al. 2016).

The most similar known virus to AGV2 is the insect virus EeV, a member of the family Permutotetraviridae (ICTV 2015; Zeddam et al. 2010), but with only $33 \%$ amino acid sequence identity and at most $38 \%$ coverage to AGV2. Based on the relationships in the tree in Figure 3A, AGV2 is likely to be the first member of a new virus family. Moreover, their virion proteins differ. EeV encodes two virion proteins in a single $\mathrm{ORF}$, one of which is a 7-kDa fragment that becomes cleaved from the $\mathrm{N}$-terminus of the major coat protein during virion maturation (Zeddam et al. 2010). In contrast, ORF2 of AGV2, predicted to encode the viral coat protein, is followed immediately by ORF3 in the same reading frame, separated by a single stop codon. We predict that ORF3 of AGV2 is translated by in-frame readthrough of the ORF2 stop codon, adding a 267 amino acid C-terminal extension to some copies of the $\mathrm{CP}$. This is because ORF3 contains a run of 9 proline codons (CCX) beginning three

TABLE 2

Virus genome assembly segments and BLASTx alignment results

\begin{tabular}{|c|c|c|c|c|c|c|c|}
\hline \multicolumn{4}{|c|}{ Sequence } & \multicolumn{4}{|c|}{ Top BLASTx hits } \\
\hline Segment & Location & Length (nt) & $\begin{array}{l}\text { Read } \\
\text { counts }\end{array}$ & Virus & $\begin{array}{l}\text { Genome } \\
\text { length (nt) }\end{array}$ & $\begin{array}{c}\text { Hit } \\
\text { length (aa) }\end{array}$ & Identity (\%) \\
\hline \multirow{2}{*}{ S1 } & Michigan & 4,838 & 38,515 & & & 631 & 33 \\
\hline & Ohio & 4,839 & 22,194 & & & 631 & 33 \\
\hline \multirow{3}{*}{ S2 } & Michigan & 9,918 & 507 & & & 1,501 & 95 \\
\hline & Ohio & 9,075 & 567 & & & 756 & 93 \\
\hline & China & 9,814 & $2,365,146$ & & & 1,021 & 94 \\
\hline S4 & China & 9,494 & $3,772,552$ & & & 485 & 90 \\
\hline S5 & China & 5,841 & 6,277 & Cotton leafroll dwarf virus & 5,865 & 638 & 95 \\
\hline \multirow[t]{4}{*}{ S6 } & lowa & 9,445 & 4,202 & Rhopalosiphum padi virus & 10,011 & 1,990 & 73 \\
\hline & Michigan & 9,443 & 4,783 & & & 1,990 & 73 \\
\hline & Ohio & 9,445 & 4,163 & & & 1,990 & 73 \\
\hline & China & 9,445 & $20,784,187$ & & & 1,990 & 73 \\
\hline
\end{tabular}


codons downstream of the major CP ORF stop codon. This resembles the sequence controlling readthrough of the CP ORF stop codon of viruses in the family Luteoviridae. In that case, a run of eight or more CCX.XXX repeats (alternating proline codons), beginning three codons downstream of the CP ORF stop codon, is required for ribosomal readthrough of the $\mathrm{CP}$ ORF stop codon (Brown et al. 1996). The resulting readthrough domain (RTD) of the luteovirid coat protein is required for transmission of the virion from within the aphid vector (in which it does not replicate) into the host plant (Brault et al. 1995; Chay et al. 1996). Thus, it is possible that the putative RTD of AGV2 CP facilitates similar interactions within the aphid, but instead facilitates infection of the aphid itself.

Segment S2. Considering that the amino acid sequence identity between S2 and ALPV is 92\%, we named S2 as ALPV-AG, a new ALPV isolate from Aphis glycines. Although we cannot conclusively state that ALPV-AG replicates in soybean aphid, the high number of reads of ALPV-AG, as well as RhPV (S4) (both known to infect other aphid species), especially in the Chinese sample, suggest that these sequences represent replicating viral genomes. In support of this, the host range of ALPV seems to be rather wide, because several other new isolates of ALPV have been discovered in other insect species: ALPV-AP from the pea aphid, Acyrthosiphon pisum, and the closely related ALPV-AM, from honey bee (Liu et al. 2014). The ALPV-AP genome is $88 \%$ identical to ALPV-AG from the sample from China, and is $86 \%$ identical to ALPV-AG from the Michigan sample.

ALPV was isolated first from R. padi, in which it can be much more pathogenic than is RhPV. ALPV can accumulate to much higher concentrations than RhPV in $R$. padi, and it can limit $R$. padi populations in the field (Williamson et al. 1988). ALPV has been associated with rapid decline of populations of the major aphid species colonizing small grains under natural conditions (Laubscher and von Wechmar 1992, 1993). The ALPV-An strain is highly pathogenic to Myzus persicae (Dombrovsky and Luria 2013). However, no acute pathogenicity was observed in ALPV-AP infection of Acyrthosiphon pisum under ideal rearing conditions (Liu et al. 2014), or by ALPV-AG presence in Aphis glycines. This suggests that the ALPVs vary in biological properties such as pathogenicity. Several ALPV-like viruses have been identified in organisms other than aphids, ranging from bat fecal samples to honey bees (Dombrovsky and Luria 2013; Ge et al. 2012; Granberg et al. 2013; Ravoet et al. 2013; Runckel et al. 2011; Williamson
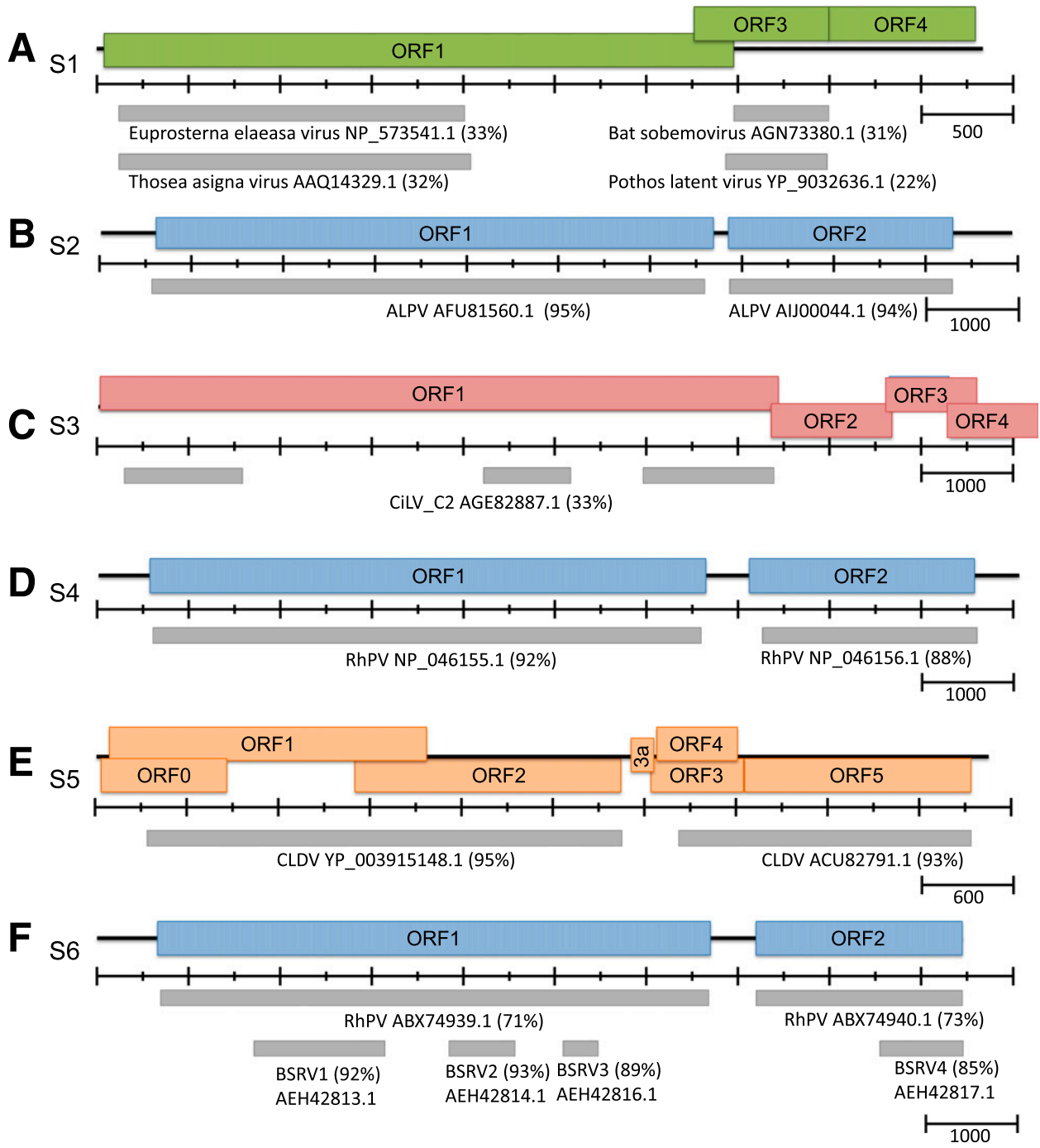

Fig. 2. Genome organizations of the assembled virus segments $S 1$ through $S 6$ aligned with most closely related sequence fragments in GenBank. Genomes are color coded based on taxonomic class as shown in Figure 3. Most closely related known viral sequences (gray bars over their GenBank accession numbers) were positionally mapped to the genomes of S1 to S6 (A to F) based on the percent identities of the associated amino acid sequences (in parentheses) to the corresponding reference genome proteins. 

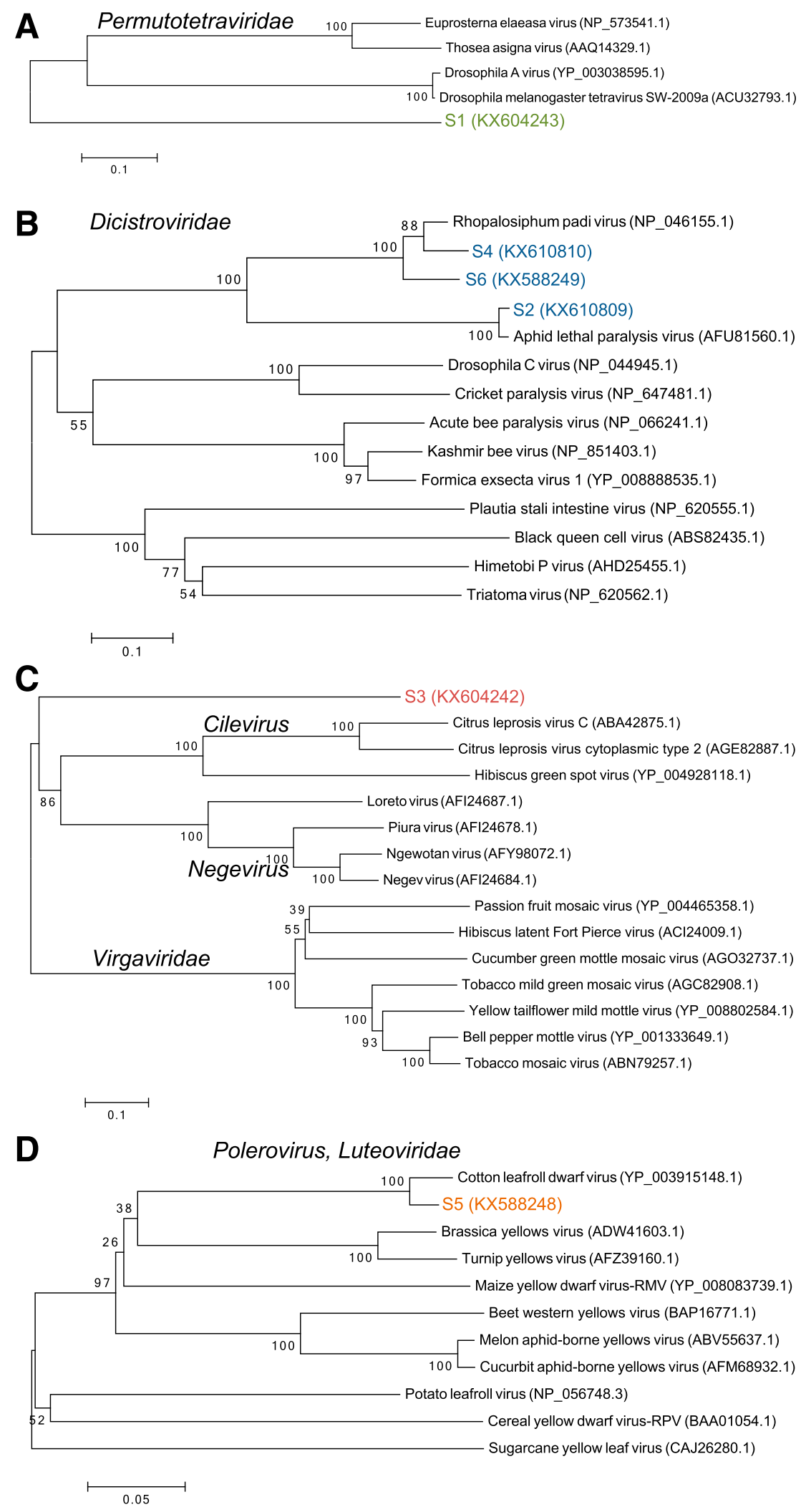

Fig. 3. Phylogenetic analysis of assembled virus genome segments. Phylogenetic trees (color coded as in Figure 2) comparing RNA-dependent RNA polymerase of A, S1 with members of the family Permutotetraviridae and related viruses; B, S2, S4, and S6 with representative members of the family Dicistroviridae; C, S3 with representative members of the genera Cilevirus and Negevirus and the family Virgaviridae; and D, S5 with representative members of the genus Polerovirus within the family Luteoviridae. Phylogenetic trees were constructed by using the maximum likelihood method in the MEGA6 program (Tamura et al. 2011) with 500 bootstrap replicates. The percentage of replicate trees in which the associated taxa clustered together in the bootstrap test is shown next to the branch points. The tree is drawn to scale, with branch lengths proportional to the number of substitutions per site (bar). GenBank accession numbers are shown for each viral sequence. 
et al. 1988). These results indicate that ALPV is highly adaptable to many insect species.

Segment S3. S3, from the Ohio sample, has little identity to any virus, but ORF1 has partial and distant identity (33\%) to ORF1 (encoding the RdRp) of the plant virus CiLV-C2. S3 differs from CiLV-C2 by containing four ORFs on a genomic RNA of at least 10,147 nt, whereas CiLV-C2 has a bipartite genome, with the 8,717 nt RNA1 encoding the RdRp and coat protein in two ORFs, and the remaining ORFs encoded on the 4,989 nt RNA2 (Roy et al. 2013). In the phylogenetic tree (Fig. 3C), two viral clades are distantly related to S3. One clade contains all plant viruses belonging to Cilevirus and Higrevirus genera. The other clade contains the insect virus genus Negevirus, which consists of six insect viruses of mosquitoes and phlebotomine sand flies (Vasilakis et al. 2013). It is clear that S3 is so distant from the above viruses that it must belong to a new family. Whether it is a plant virus or an insect virus is unknown. We designate S3, perhaps temporarily, as Aphis glycines virus 3 (AGV3).

Segment S4. Both ORFs of S4 have $>90 \%$ amino acid identity to RhPV, hence S4 is not a new virus, but a strain of RhPV, which we designate as RhPV-AG, a new RhPV isolate from Aphis glycines. Until now, RhPV was known to infect only cereal aphids, including R. padi (bird cherry-oat aphid) (D'Arcy et al. 1981), R. rufiabdominalis (rice root aphid) (D'Arcy et al. 1981), Diuraphis noxia (Russian wheat aphid) (von Wechmar and Rybicki 1981), R. maidis (green corn aphid), Acyrthosiphum dirhodum (grain aphid) (Williamson et al. 1989), and Schizaphis graminum (greenbug) (D'Arcy et al. 1981; Gildow and D'Arcy 1988).

Segment S5. S5 appeared only in the sample from China, sharing 93\% amino acid identity with the plant virus CLRDV. Thus, S5 is an isolate of CLRDV. CLRDV is the causal agent of cotton blue disease (CBD) and is a polerovirus (Luteoviridae) known to be transmitted only by Aphis gossypii Glover (Correa et al. 2005; Distefano et al. 2010). Luteovirids are transmitted by the aphid in a circulative, nonpropagative manner. CLRDV-infected cotton (Gossypium hirsutum) plants show a moderate to severe stunting phenotype due to shortening of internodes, leaf rolling, vein yellowing and intensive dark green color of the foliage (Cauquil and Vaissayre 1971). CBD has been found only in South America, Africa and Asia (Cauquil 1977), which explains why we did not find it in aphids collected in the Midwest, far from any cotton growing region. Although found in Thailand and other southeast Asian countries, CLRDV has not been reported in China (M. Sharman, personal communication). Presence of CLRDV in soybean aphid suggests the possibility that soybean aphid may serve as a vector of CLRDV. For the soybean aphid to have acquired CLRDV, we speculate that either CLRDV infects soybean and/or soybean aphid colonizes cotton. However, soybean aphid has been reported to feed poorly or not at all on cotton (Han and Yan 1995). Thus, perhaps CLRDV can infect soybean in China, via the vector Aphis gossypii, which has a wide host range that includes soybean. However, to our knowledge, infection of soybean by CLRDV has not been reported. Alternatively, CLRDV may be particularly stable and widespread and simply came into contact with the soybean aphid sample by other means. Consistent with this possibility, a meta-analysis of small RNA sequencing libraries from many organisms identified a CLRDV sequence fragment in a mouse (Mus musculus) small RNA dataset (Wang et al. 2014). However, given that CLRDV is stable and abundant enough to yield sufficient reads for complete genome assembly, we surmise that CLRDV is likely to be vectored by the soybean aphid in China.

Segment S6. S6 shares $71 \%$ amino acid identity with RhPV, and has the same genome organization, placing it in the family Dicistroviridae. Interestingly, S6 has highest amino acid identity (ranging from 85 to 93\%) with the four contigs attributed to BSRV from honey bee (Fig. 2F). Those non-overlapping contigs were obtained by deep sequencing of viruses isolated from honey bee (Runckel et al. 2011). While it wasn't clear whether all four contigs came from the same virus, given that our full-length genome assembly has high homology to all four contigs, we have apparently completed the genome sequence of BSRV to which these four contigs belong. Which hosts BSRV actually infects are unknown, but given the high number of reads we obtained (over 4,500 reads per sample), the isolate represented by S6 is likely to infect soybean aphid.

In summary, our results reveal that a variety of viruses associate with soybean aphid, but it is not certain which actually replicate in this host. Given the high number of reads for these viruses in the Chinese isolate (Table 2), it is likely that the dicistroviruses ALPV, RhPV, and BSRV infect and replicate in the soybean aphid. Moreover, ALPV and RhPV are known aphid viruses. However, the Chinese isolate may be biased, as the long incubation at ambient temperature of the aphid carcasses led to degradation of most RNAs (including AGV2, above), leaving those RNAs in the highly stable dicistrovirus virions to dominate the pool of sequenceable RNAs. It is unclear whether the cilevirus-like virus (AGV3) infects the soybean aphid or is simply associated with it. We know that CLRDV does not replicate in soybean aphid because much research has shown that poleroviruses (like all of the Luteoviridae) do not replicate in aphids (Gray and Gildow 2003). This may explain why the number of reads of CLRDV is three orders of magnitude less than those of the dicistroviruses in the Chinese sample.

We assembled only six full viral genomes, but there are still many small contigs in each sample that hit a wide range of viral genomes. However, most of those contigs have short hit lengths ( $<100 \mathrm{nt})$ and low read count $(<50)$ due to several possible reasons: (i) random sequence matching because the hit length is short even with an e-value less than $1 \mathrm{E}-10$, the contig still has a low identity to the viral genome that was "hit"; (ii) samples may have been contaminated during the isolation; and (iii) some noncirculative virions remain on the surface of aphids.

We emphasize that this study by no means reveals the whole aphid virome. It is noteworthy that we detected no plant viruses already known to be transmitted by soybean aphid, such as AMV, SbDV, or SMV. This may be because three of our four samples were from aphids that had been maintained in growth chambers, under relatively diseasefree conditions. These results provide baseline sequences for comparison of future virome surveys to better understand the possible plant and insect viruses that aphids may carry. Such viruses may have potential for use as biological control agents. Viruses such as BSRV may play roles in agroecosystems by infecting both pest (aphids) and beneficial insects (honey bees). This viromics approach also may inform quarantine and containment policies, as deep sequencing reveals that many viruses may be far more widespread than previously thought. Finally, the discovery reported here of three genomes of previously unknown virus families reveals the ever-increasing possibilities of virus diversity that were unimagined before the advent of deep sequencing.

\section{ACKNOWLEDGMENTS}

We thank X. Sun, Wuhan University; A. Michel, Ohio State University; D. Landis, Michigan State University; and M. O'Neal, Iowa State University for providing aphids from China, Ohio, Michigan, and Iowa, respectively. This project was funded by the Iowa Soybean Association, and the Iowa State University Plant Sciences Institute. This journal paper of the Iowa Agriculture and Home Economics Experiment Station, Ames, IA, Project 3708, was supported in part by Hatch Act and State of Iowa funds. 


\section{LITERATURE CITED}

Belliure, B., Janssen, A., and Sabelis, M. W. 2008. Herbivore benefits from vectoring plant virus through reduction of period of vulnerability to predation. Oecologia 156:797-806.

Bol, J. F. 2003. Alfalfa mosaic virus: Coat protein-dependent initiation of infection. Mol. Plant Pathol. 4:1-8.

Bonning, B. C., and Miller, W. A. 2010. Dicistroviruses. Annu. Rev. Entomol. 55:129-150.

Brault, V., Uzest, M., Monsion, B., Jacquot, E., and Blanc, S. 2010. Aphids as transport devices for plant viruses. C. R. Biol. 333:524-538.

Brault, V., Van den Heuvel, J. F. J. M., Verbeek, M., Ziegler-Graff, V., Reutenauer, A., Herrbach, E., Garaud, J. C., Guilley, H., Richards, K., and Jonard, G. 1995. Aphid transmission of beet western yellows luteovirus requires the minor capsid read-through protein P74. EMBO J. 14: 650-659.

Brown, C. M., Dinesh-Kumar, S. P., and Miller, W. A. 1996. Local and distant sequences are required for efficient readthrough of the barley yellow dwarf virus PAV coat protein gene stop codon. J. Virol. 70:5884-5892.

Cassone, B. J., Michel, A. P., Stewart, L. R., Bansal, R., Mian, M. A., and Redinbaugh, M. G. 2014. Reduction in fecundity and shifts in cellular processes by a native virus on an invasive insect. Genome Biol. Evol. 6: 873-885.

Casteel, C. L., Yang, C., Nanduri, A. C., De Jong, H. N., Whitham, S. A., and Jander, G. 2014. The NIa-Pro protein of Turnip mosaic virus improves growth and reproduction of the aphid vector, Myzus persicae (green peach aphid). Plant J. 77:653-663.

Cauquil, J. 1977. Etudes sur une maladie d'origine virale du cotonnier: La maladie bleue. Coton Fibres Tropicales 32:259-278.

Cauquil, J., and Vaissayre, M. 1971. La "maladie bleue" du cotonnier en Afrique: Transmission de cotonnier a cotonnier par Aphis gossypii Glover. Coton Fibres Tropicales 26:462-466.

Chay, C. A., Gunasinge, U. B., Dinesh Kumar, S. P., Miller, W. A., and Gray, S. M. 1996. Aphid transmission and systemic plant infection determinants of barley yellow dwarf luteovirus-PAV are contained in the coat protein readthrough domain and 17-kDa protein, respectively. Virology 219:57-65.

Correa, R. L., Silva, T. F., Simoes-Araujo, J. L., Barroso, P. A., Vidal, M. S., and Vaslin, M. F. 2005. Molecular characterization of a virus from the family Luteoviridae associated with cotton blue disease. Arch. Virol. 150: 1357-1367.

Costantino, D. A., Pfingsten, J. S., Rambo, R. P., and Kieft, J. S. 2008. tRNAmRNA mimicry drives translation initiation from a viral IRES. Nat. Struct. Mol. Biol. 15:57-64.

D’Arcy, C. J., Burnett, P. A., and Hewings, A. D. 1981. Detection, biological effects, and transmission of a virus of the aphid Rhopalosiphum padi. Virology 114:268-272.

Damsteegt, V. D., Stone, A. L., Kuhlmann, M., Gildow, F. E., Domier, L. L., Sherman, D. J., Tian, B., and Schneider, W. L. 2011. Acquisition and transmissibility of U.S. Soybean dwarf virus isolates by the soybean aphid, Aphis glycines. Plant Dis. 95:945-950.

Distefano, A. J., Bonacic Kresic, I., and Hopp, H. E. 2010. The complete genome sequence of a virus associated with cotton blue disease, cotton leafroll dwarf virus, confirms that it is a new member of the genus Polerovirus. Arch. Virol. 155:1849-1854.

Dombrovsky, A., and Luria, N. 2013. The Nerium oleander aphid Aphis nerii is tolerant to a local isolate of Aphid lethal paralysis virus (ALPV). Virus Genes 46:354-361.

Donaldson, J. R., and Gratton, C. 2007. Antagonistic effects of soybean viruses on soybean aphid performance. Environ. Entomol. 36:918-925.

Fiebig, M., Poehling, H. M., and Borgemeister, C. 2004. Barley yellow dwarf virus, wheat, and Sitobion avenae: A case of trilateral interactions. Entomol. Exp. Appl. 110:11-21.

Ge, X., Li, Y., Yang, X., Zhang, H., Zhou, P., Zhang, Y., and Shi, Z. 2012. Metagenomic analysis of viruses from bat fecal samples reveals many novel viruses in insectivorous bats in China. J. Virol. 86:4620-4630.

Gildow, F. E., and D'Arcy, C. J. 1988. Barley and oats as reservoirs for an aphid virus and the influence on barley yellow dwarf virus transmission. Phytopathology 78:811-816.

Gildow, F. E., and D'Arcy, C. J. 1990. Cytopathology and experimental host range of Rhopalosiphum padi virus, a small isometric RNA virus infecting cereal grain aphids. J. Invertebr. Pathol. 55:245-257.

Granberg, F., Vicente-Rubiano, M., Rubio-Guerri, C., Karlsson, O. E., Kukielka, D., Belak, S., and Sanchez-Vizcaino, J. M. 2013. Metagenomic detection of viral pathogens in Spanish honeybees: Co-infection by aphid lethal paralysis, Israel acute paralysis and Lake Sinai viruses. PLoS ONE 8:e57459.

Gray, S., and Gildow, F. E. 2003. Luteovirus-aphid interactions. Annu. Rev. Phytopathol. 41:539-566.

Han, X., and Yan, F. 1995. Stylet penetration behavior of soybean aphid, Aphis glycines, on host and non-host plants. Acta Entomol. Sin. 38:278-283.

ICTV. 2015. Virus Taxonomy: 2015 Release. International Committee on Taxonomy of Viruses.

Jayaram, C., Hill, J. H., and Miller, W. A. 1991. Nucleotide sequences of the coat protein genes of two aphid-transmissible strains of Soybean mosaic virus. J. Gen. Virol. 72:1001-1003.

Kim, C. H., Schaible, G., Garrett, L., Lubowski, R., and Lee, D. 2008. Economic impacts of the U.S. soybean aphid infestation: A multi-regional competitive dynamic analysis. Agric. Res. Econ. Rev. 37:227-242.

Lacroix, C., Jolles, A., Seabloom, E. W., Power, A. G., Mitchell, C. E., and Borer, E. T. 2014. Non-random biodiversity loss underlies predictable increases in viral disease prevalence. J. R. Soc. Interface 11:20130947.

Laubscher, J. M., and von Wechmar, M. B. 1992. Influence of aphid lethal paralysis virus and Rhopalosiphum padi virus on aphid biology at different temperatures. J. Invertebr. Pathol. 60:134-140.

Laubscher, J. M., and von Wechmar, M. B. 1993. Assessment of aphid lethal paralysis virus as an apparent population growth-limiting factor in grain aphids in the presence of other natural enemies. Biocontrol Sci. Technol. 3:455-466.

Liu, S., Vijayendran, D., and Bonning, B. C. 2011. Next generation sequencing technologies for insect virus discovery. Viruses 3:1849-1869.

Liu, S., Vijayendran, D., Carrillo-Tripp, J., Miller, W. A., and Bonning, B. C. 2014. Analysis of new aphid lethal paralysis virus (ALPV) isolates suggests evolution of two ALPV species. J. Gen. Virol. 95:2809-2819.

Liu, S., Vijayendran, D., Chen, Y., and Bonning, B. C. 2016. Aphis glycines virus 2, a novel insect virus with a unique genome structure. Viruses 8:315.

Macedo, T. B., Bastos, C. S., Higley, L. G., Ostlie, K. R., and Madhavan, S. 2003. Photosynthetic responses of soybean to soybean aphid (Homoptera: Aphididae) injury. J. Econ. Entomol. 96:188-193.

Malmstrom, C. M., McCullough, A. J., Johnson, H. A., Newton, L. A., and Borer, E. T. 2005. Invasive annual grasses indirectly increase virus incidence in California native perennial bunchgrasses. Oecologia 145:153-164.

Mann, K. S., and Dietzgen, R. G. 2014. Plant rhabdoviruses: New insights and research needs in the interplay of negative-strand RNA viruses with plant and insect hosts. Arch. Virol. 159:1889-1900.

McIntyre, J. L., Dodds, J. A., and Hare, J. D. 1981. Effects of localized infections of Nicotiana tabacum by Tobacco mosaic virus on systemic resistance against diverse pathogens and an insect. Phytopathology 71:297-301.

Mordecai, E. A., Gross, K., and Mitchell, C. E. 2016. Within-host niche differences and fitness trade-offs promote coexistence of plant viruses. Am. Nat. 187:E13-E26.

Mowry, T. M. 1994. Russian wheat aphid (Homoptera, Aphididae) survival and fecundity on Barley yellow dwarf virus-infected wheat resistant and susceptible to the aphid. Environ. Entomol. 23:326-330.

Ng, J. C., and Falk, B. W. 2006. Virus-vector interactions mediating nonpersistent and semipersistent transmission of plant viruses. Annu. Rev. Phytopathol. 44:183-212.

Ragsdale, D. W., Voegtlin, D. J., and O’Neil, R. J. 2004. Soybean aphid biology in North America. Ann. Entomol. Soc. Am. 97:204-208.

Ravoet, J., Maharramov, J., Meeus, I., De Smet, L., Wenseleers, T., Smagghe, G., and de Graaf, D. C. 2013. Comprehensive bee pathogen screening in Belgium reveals Crithidia mellificae as a new contributory factor to winter mortality. PLoS ONE 8:e72443.

Roy, A., Choudhary, N., Guillermo, L. M., Shao, J., Govindarajulu, A., Achor, D., Wei, G., Picton, D. D., Levy, L., Nakhla, M. K., Hartung, J. S., and Brlansky, R. H. 2013. A novel virus of the genus Cilevirus causing symptoms similar to citrus leprosis. Phytopathology 103:488-500.

Runckel, C., Flenniken, M. L., Engel, J. C., Ruby, J. G., Ganem, D., Andino, R., and DeRisi, J. L. 2011. Temporal analysis of the honey bee microbiome reveals four novel viruses and seasonal prevalence of known viruses, Nosema, and Crithidia. PLoS ONE 6:e20656.

Ryabov, E. V. 2007. A novel virus isolated from the aphid Brevicoryne brassicae with similarity to Hymenoptera picorna-like viruses. J. Gen. Virol. 88: 2590-2595.

Ryabov, E. V., Keane, G., Naish, N., Evered, C., and Winstanley, D. 2009. Densovirus induces winged morphs in asexual clones of the rosy apple aphid, Dysaphis plantaginea. Proc. Natl. Acad. Sci. USA 106:8465-8470.

Smirnova, E., Firth, A. E., Miller, W. A., Scheidecker, D., Brault, V., Reinbold, C., Rakotondrafara, A. M., Chung, B. Y., and Ziegler-Graff, V. 2015. Discovery of a small non-AUG-initiated ORF in poleroviruses and 
luteoviruses that is required for long-distance movement. PLoS Pathog. 11: e1004868.

Tamura, K., Peterson, D., Peterson, N., Stecher, G., Nei, M., and Kumar, S. 2011. MEGA5: Molecular evolutionary genetics analysis using maximum likelihood, evolutionary distance, and maximum parsimony methods. Mol. Biol. Evol. 28:2731-2739.

Teixeira, M., Sela, N., Ng, J., Casteel, C. L., Peng, H.-C., Bekal, S., Girke, T., Ghanim, M., and Kaloshian, I. 2016. A novel virus from Macrosiphum euphorbiae with similarities to members of the family Flaviviridae. J. Gen. Virol. 97:1261-1271.

van den Heuvel, J. F., Hummelen, H., Verbeek, M., Dullemans, A. M., and van der Wilk, F. 1997. Characteristics of Acyrthosiphon pisum virus, a newly identified virus infecting the pea aphid. J. Invertebr. Pathol. 70: 169-176.

van Munster, M., Dullemans, A. M., Verbeek, M., van den Heuvel, J. F., Reinbold, C., Brault, V., Clerivet, A., and van der Wilk, F. 2003.

Characterization of a new densovirus infecting the green peach aphid Myzus persicae. J. Invertebr. Pathol. 84:6-14.

Vasilakis, N., Forrester, N. L., Palacios, G., Nasar, F., Savji, N., Rossi, S. L., Guzman, H., Wood, T. G., Popov, V., Gorchakov, R., Gonzalez, A. V.,
Haddow, A. D., Watts, D. M., da Rosa, A. P., Weaver, S. C., Lipkin, W. I., and Tesh, R. B. 2013. Negevirus: A proposed new taxon of insect-specific viruses with wide geographic distribution. J. Virol. 87:2475-2488.

von Wechmar, M. B., and Rybicki, E. P. 1981. Aphid transmission of three viruses causes Free State streak disease. S. Afr. J. Sci. 77:488-492.

Wang, Y., Wang, H., Xu, K., Ni, P., Zhang, H., Ma, J., Yang, H., and Xu, F. 2014. A survey of overlooked viral infections in biological experiment systems. PLoS ONE 9:e105348.

Williamson, C., Rybicki, E. P., Kasdorf, G. G. F., and von Wechmar, M. B. 1988. Characterization of a new picorna-like virus isolated from aphids. J. Gen. Virol. 69:787-795.

Williamson, C., Wechmar, M. B. V., and Rybicki, E. P. 1989. Further characterization of Rhopalosiphum padi virus of aphids and comparison of isolates from South Africa and Illinois. J. Invertebr. Pathol. 54:85-96.

Zeddam, J. L., Gordon, K. H., Lauber, C., Alves, C. A., Luke, B. T., Hanzlik, T. N., Ward, V. K., and Gorbalenya, A. E. 2010. Euprosterna elaeasa virus genome sequence and evolution of the Tetraviridae family: Emergence of bipartite genomes and conservation of the VPg signal with the dsRNA Birnaviridae family. Virology 397:145-154. 